\title{
Multifocal facial Rosai Dorfman disease with postsurgical recurrence
}

\author{
Ishita Sen ${ }^{1}$, Subhadeep Chowdhury ${ }^{2}$, Tithi Debnath ${ }^{2 *}$
}

\author{
${ }^{1}$ Department of ENT, Suri Superspeciality Hospital, Birbhum, West Bengal, India \\ ${ }^{2}$ Department of ENT, R. G. Kar Medical College and Hospital, West Bengal, India
}

Received: 23 May 2021

Accepted: 15 July 2021

*Correspondence:

Tithi Debnath,

E-mail: tithi_debnath@rediffmail.com

Copyright: (C) the author(s), publisher and licensee Medip Academy. This is an open-access article distributed under the terms of the Creative Commons Attribution Non-Commercial License, which permits unrestricted non-commercial use, distribution, and reproduction in any medium, provided the original work is properly cited.

\begin{abstract}
Rosai Dorfman disease (RDD) is a histiocytic proliferative disorder with massive painless lymphadenopathy that though is rare yet has been reported more than seldom. Our report aimed to emphasis on a case with multifocal facial involvement with postsurgical recurrence, which had not yet been reported and an approach to alleviate the patient's symptoms and avert a fatal outcome. This study was a case report and literature review. A 19 year old female presented with swelling involving right sided cheek, periorbital region and bilateral nasal cavity with palatal perforation for last 6 months. She had a past history of debulking of orbital RDD. Contrast enhanced computed tomography revealed an extraconal mass of the right orbit and left nasal mass extending to the nasopharynx. Biopsy taken from the nasal masses showed RDD on histopathology and immunohistochemistry. Though the disease was self-resolving, this patient required radiotherapy as debulking was not sufficient for its multifocal presentation and was followed by palatal reconstruction
\end{abstract}

Keywords: Sinus histiocytosis, Multifocal, Postsurgical recurrence, Emperipolesis, Radiotherapy

\section{INTRODUCTION}

RDD also known as sinus histiocytosis with massive lymphadenopathy is a rare histiocytic proliferative disorder of unknown etiology. It is characterised by massive painless lymphadenopathy due to expansion of sinuses infiltrated with benign non-langerhans histiocytes and plasma cells. ${ }^{1}$ It was first described by Destombes in 1965 and it was Rosai and Dorfman who published a paper on sinus histiocytosis with massive lymphadenopathy in 1969 and characterised it as a distinct clinicopathological entity. ${ }^{2}$ It occurs in young adults and the mean age of diagnosis is 20 years occurring most commonly in males. ${ }^{3,4}$ The patients also present with fever and leucocytosis. It has predilection for lymph nodes of the head and neck region. Extranodal involvement has been found to occur in up to $40 \%$ of patients with the disease. Skin is the most common extranodal site of involvement. In the face, skin is the most common structure involved followed by nose, paranasal sinuses, orbit, salivary glands and rarely masticator space. Other organs involved are central nervous system, upper respiratory tract, gastrointestinal tract, liver and spleen. ${ }^{2-6}$ There is no clear known etiology. Associations with infectious agents and immune dysfunction have been found. Human herpes virus-6, parvovirus B19, cytomegalovirus have been speculated to cause histiocytic proliferation. A study also showed the association of autoimmune disease with disordered apoptotic signaling pathway in stimulating histiocytic proliferation. ${ }^{2,3,5}$ A recent few studies have shown NRAS, KRAS, ARAS, MAP2K1 mutations in its origin. ${ }^{7}$ Our case reported outlays RDD in a 19 year old female with skin, orbital and nasal involvement with recurrence and an approach to its management.

\section{CASE REPORT}

A 19 year old female presented with swelling involving right sided cheek and periorbital region for 6 months, 
gradually increasing in size with associated anosmia, nasal obstruction, epiphora, dysphagia, recurrent fever and occasional epistaxis. She had a past history of debulking of right orbital mass 4 years back and swelling involving right parotid region 1 year back that resolved with discharge from the lesion. The debulked orbital mass had findings consistent with RDD on HPE (histopathological pathogical examination). On examination, the skin over the right side of face was reddish, swollen and non-tender. There was nasal tip deviation to the left and obliteration of the right nasolabial fold (Figure 1). She also had bulging of hard palate more on the right side with palatal perforation. Visual acuity and other cranial nerve examination were normal. The patient had no palpable lymph nodes. DNE (diagnostic nasal endoscopy) showed a swelling involving the right nasal cavity lined by skin and mucosa with smooth surface, arising from the lateral nasal wall. In the left nasal cavity, there was a proliferative growth involving the inferior turbinate with a gross DNS to the left (Figure 2). CECT (contrast enhanced computed tomography) scan of the face and neck showed two separate enhancing soft tissue densities in both right and left nasal cavities; the left nasal mass extended into the nasopharynx compromising nasopharyngeal airway; DNS to left and pansinusitis (Figure 3). Extraconal heterogenous soft tissue density was also seen in right orbit extending to cheek, upper jaw, nasal bridge, upper and lower eyelids. No cervical lymph node or neck lesion was noted. Biopsy of the nasal masses were taken by sublabial incision and sent for HPE and immunohistochemistry. HPE showed abundant histiocytes with pale eosinophilic cytoplasm, mildly atypical round vesicular nuclei with emperipolesis or lymphophagocytosis. Immunohistochemistry had S100 staining positive. The patient was given a course of radiotherapy for 2 weeks and had a shrinking of the nasal, orbital masses and resolution of skin swelling. The patient was initially kept on feeding jejunostomy to prevent nasal regurgitation due to palatal perforation and later underwent palatal flap reconstruction in the department of plastic surgery. The patient followed up in the ENT OPD after 6 months of completion of radiotherapy and showed no recurrence on clinical examination and DNE.

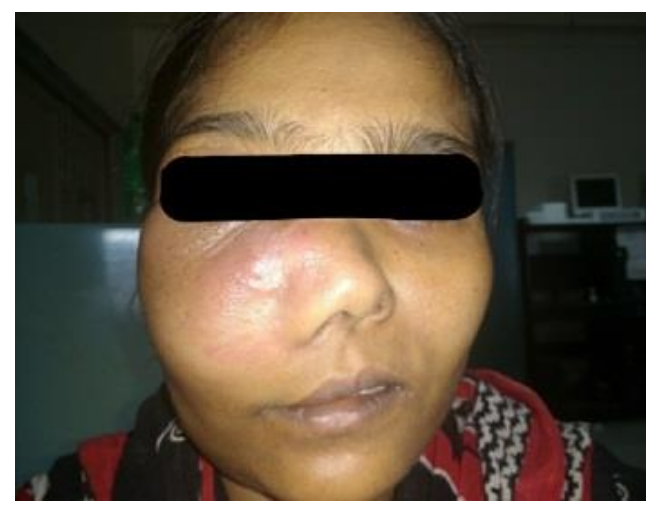

Figure 1: Clinical picture on presentation.

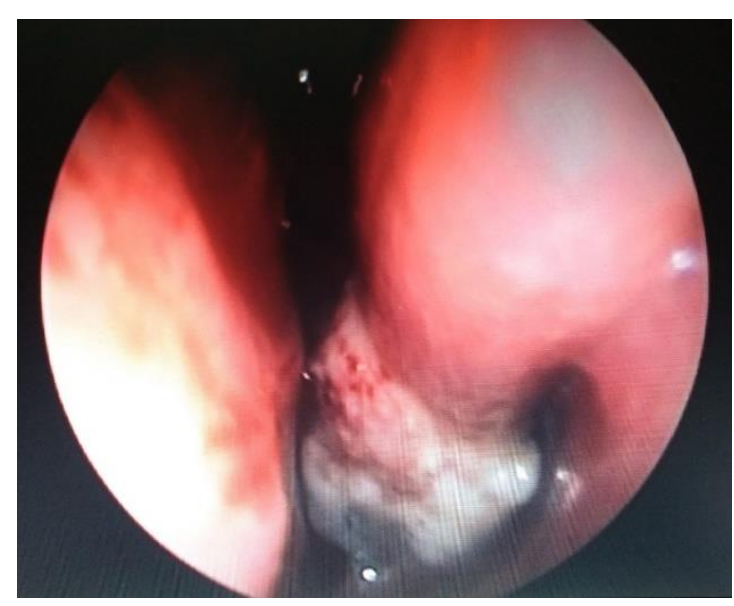

Figure 2: Diagnostic Nasal Endoscopy showing proliferative growth involving the inferior turbinate with a gross DNS to the left.

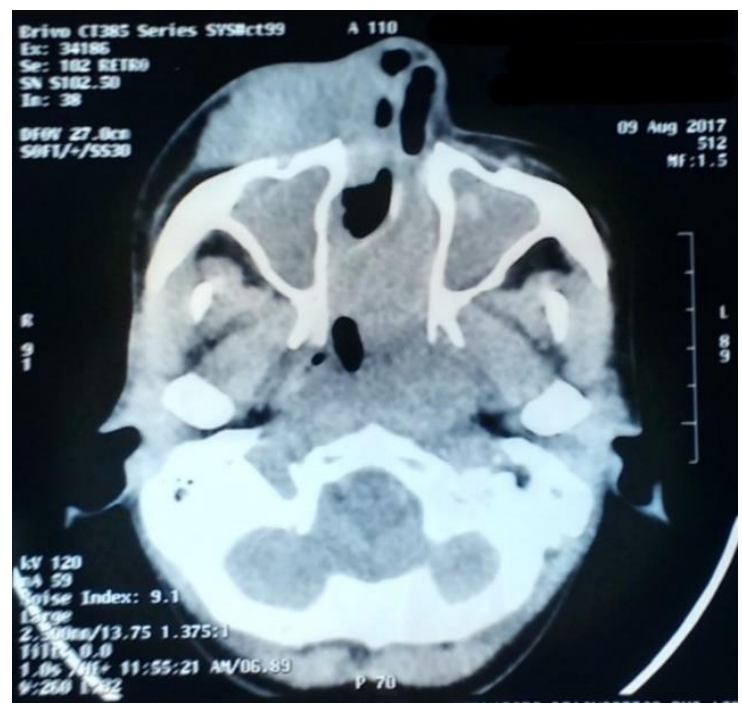

Figure 3: CECT of Face and neck.

\section{DISCUSSION}

The hallmark of RDD is bilateral lymphadenopathy mainly involving the cervical region with a few patients presenting with fever and malaise. Cutaneous RDD is mainly seen in the head and neck region, more commonly occurring in females with average age of diagnosis being 45 years. $^{2-6}$ Within the head and neck region, nose is the most commonly affected site. Central nervous system involvement commonly mimics other central nervous system tumours. ${ }^{2,5}$ Human face is divided into buccal, infraorbital, mental, nasal, orbital, parotid and zygomatic regions and comprises of skin, fat pad, muscles, nerves, vessels, facial bones, eyes, nose, ears, lacrimal glands, parotids. Having an important role to play in respiration, smell, speech, hearing, vision and deglutition, involvement of facial structures especially in multiple is a matter to consider. Facial RDD most commonly presents with cutaneous involvement alone, presenting as papules or knots under the skin to palpable masses with or 
without satellite lesions..$^{2-6}$ Nose and paranasal sinuses involvement presents as nasal obstruction, rhinitis and epistaxis, anosmia. ${ }^{8}$ When involving the orbital region, proptosis, restricted eye movement, periorbital edema, epiphora and diminished visual acuity are common. ${ }^{9,10}$ Though the reported cases of multifocal RDD have described lesions involving various parts of the body nodal, extranodal or both, yet multifocal lesions involving only the face has not been reported. Recurrence isn't unusual. A study in 2011 reported $54.8 \%$ cases to have recurrent, persistent or progressive course. ${ }^{13} \mathrm{~A}$ comprehensive study of all extranodal soft tissue RDD cases from 1988 to 2011 revealed only one recurrence post excision. Till date only two postsurgical soft tissue recurrences have been reported. ${ }^{14}$ Our case being multifocal while solely involved facial structures and recurrent post-excision making it unusual.

The diagnosis was based on histology and immunohistochemistry. Fine needle aspiration cytology from the involved lymph node or HPE of the excised lesion aided in the diagnosis. The most common histological finding was proliferation of large histiocytes with abundant pale eosinophilic cytoplasm exhibiting emperipolesis that is non-destructive phagocytosis of lymphocytes, erythrocytes, plasma cells within another cell such as a histiocyte. Immunohistochemistry reveals positive CD68 and S100. ${ }^{1,2,5}$ Other laboratory findings include raised erythrocyte sedimentation rate, hypergammaglobulinemia. ${ }^{3,5}$ Imaging of RDD generally gave non-specific findings resembling malignant disease. $^{6}$

RDD usually resolves spontaneously especially, classic RDD with lymph node involvement. Only a few require treatment. ${ }^{5,8}$ Steroids are usually used as the first line of treatment. Oka et al reported that prolonged course of low dose prednisolone is effective in RDD with skin and lymph node involvement with respiratory obstruction. ${ }^{11}$ Radiotherapy has a role in palliation, for preserving vital organ function in cases orbital, airway and central nervous system involvement. ${ }^{2}$ Chemotherapeutic agents like methotrexate, 6-mercaptopurine, cladribine or vinorelbine plus methotrexate have been found to be effective in some cases. Interferon treatment has also been reported. ${ }^{3}$ Surgery is reserved for preserving organ function or in life threatening situations like decompressing the head and neck region to maintain airway patency, to reduce raised intraocular pressure or to open the paranasal sinuses. ${ }^{7}$ There was no definite treatment guideline till in a study in 2018, management algorithm of RDD was proposed. It emphasized on next generation sequencing for MAPK pathway mutations and targeted therapy with MEK inhibitor for severe and refractory cases. ${ }^{7}$ Trametinib, a MEK inhibitor has been demonstrated to be efficient in many cases irrespective of molecular profile. ${ }^{12}$ The case in our report though had a history of spontaneous resolution of previous lesions, required radiotherapy as debulking was not appropriate for the multifocal presentation of the disease and a surgical reconstruction later. Trametinib, not being readily available in the oncology department of our hospital could not be utilised in the treatment.

\section{CONCLUSION}

RDD is a rare and interesting disease. The unique characteristic being emperipolesis. The involvement of multiple facial structures at the same time without any lymphadenopathy and recurrence of orbital lesion even after debulking makes our case the only such case to be reported.

\section{Funding: No funding sources Conflict of interest: None declared Ethical approval: Not required}

\section{REFERENCES}

1. Agarwal A, Pathak S, Gujral S. Sinus histiocytosis with massive lymphadenopathy-a review of seven cases. Indian J Pathol Microbiol. 2006;49(4):50915.

2. Dalia S, Sagatys E, Sokol L, Kubal T. RosaiDorfman disease: tumor biology, clinical features, pathology and treatment. Cancer Control. 2014;21(4):322-7.

3. Ha H, Kim KH, Ahn YJ, Kim JH, Kim JE, Yoon SS. A rare case of Rosai-Dorfman disease without lymphadenopathy. Korean J Intern Med. 2016;31(4):802-4.

4. National Organization for Rare Diseases. Fact sheet: Rosai-Dorfman Disease, 2014. Available at: http://www.rarediseases.org/rare-diseaseinformation/rare-diseases/byID/1122/viewAbstract. Accessed on 8 May 2021.

5. Feriante J, Lee RT. Case report Rosai-Dorfman disease: self-resolving unilateral lymphadenopathy and a brief review of literature. Case reports Oncol Med. 2018;2018(10):1-4.

6. Mantilla JG, Goldberg-Stein SA, Wang Y. Extranodal Rosai-Dorfman disease: Clinicopathologic series of 10 patients with radiologic correlation and review of the literature. Am J Clin Pathol. 2016;145(2):211-21.

7. Abla O, Jacobsen E, Picarsic J, Krenova Z, Jaffe R, Emile $\mathrm{J}$, et al. Consensus recommendations for the diagnosis and clinical management of RosaiDorfman-Destombes disease. Blood. 2018;131(26):2877-90.

8. Sanli A, Eken M, Oktay ZA, Paksoy M, Hardal U. Rosai-Dorfman disease: sinus histiocytosis with massive lymphadenopathy. BMJ Case Rep. 2009;2009:11.

9. Narjess BR, Meriem Z, Leila K, Fafani BHH, Hachmi M. Bilateral isolated Rosai-Dorfman disease of the orbit. Can J Opthalmol. 2018;54(2):77-81.

10. Wang E, Anzai Y, Paulino A, Wong J. RosaiDorfman disease presenting with isolated bilateral 
orbital masses: report of two cases. AJNR Am J Neuroradiol. 2001;22(7):1386-8.

11. Pradhananga RB, Dangol K, Shrestha A, Baskota DK. Sinus histiocytosis with massive lymphadenopathy (Rosai-Dorfman disease): a case report and literature review. Int Arch Otorhinolaryngol. 2014;18(4):406-8.

12. Janku F, Patel BSH, Raghavan VK, Goodman A, Barnes TG, Kurzrock R. MEK inhibition with trametinib in patients with non-langerhans cell histiocytosis. Blood. 2019;134(1):2319.

13. Chen H, Zhou S, Wang S, Teng X, Fan J. Factors associated with recurrence and therapeutic strategies for sinonasal Rosai-Dorfman disease. Head Neck. 2012;34(10):1504-13.

14. Komaragiri M, Sparber LS, Santos-Zabala ML, Dardik M, Chamberlain RS. Extranodal RosaDorfman disease: a rare soft tissue neoplasm masquerading as a sarcoma. World J Surg Onc. 2013;11:63.

Cite this article as: Sen I, Chowdhury S, Debnath T. Multifocal facial Rosai Dorfman disease with postsurgical recurrence. Int J Otorhinolaryngol Head Neck Surg 2021;7:1377-80. 